\title{
Cerebral Venous Thrombosis after Ventriculoperitoneal Shunting: A Case Report
}

\author{
Teppei MAtsubarA, ${ }^{1}$ Satoshi AyuzawA, ${ }^{1}$ Tsukasa AOKI, ${ }^{2}$ Go IKEDA, ${ }^{1}$ \\ Masanari SHIIGAI, ${ }^{3}$ and Akira MATSUMURA ${ }^{1}$ \\ Departments of ${ }^{1}$ Neurosurgery and ${ }^{3}$ Radiology, \\ Graduate School of Comprehensive Human Sciences, University of Tsukuba, \\ Tsukuba, Ibaraki; \\ ${ }^{2}$ Department of Neurosurgery, Ryugasaki Saiseikai Hospital, Ryugasaki, Ibaraki
}

\begin{abstract}
Ventriculoperitoneal shunting (VPS) is a simple procedure, but there are several potential complications. We describe the first reported case of cerebral venous thrombosis (CVT) after VPS. A 69-year-old man suffering from normal pressure hydrocephalus underwent left VPS. Two months later he developed CVT and cerebral venous hemorrhage in the left frontal lobe. Computed tomography (CT) and magnetic resonance imaging (MRI) revealed the thrombus formation just adjacent to the shunt tube. One possible cause is compression of the cortical vein after brain shift and/or tension of the cortical vein due to intracranial hypotension (IH). A protein C deficiency was also detected. Surgeons should be aware that cerebral venous thrombosis can occur after VPS.
\end{abstract}

Key words: cerebral venous thrombosis, ventriculoperitoneal shunting, intracranial hypotension, protein C deficiency

\section{Introduction}

Ventriculoperitoneal shunting (VPS) is a common operation for the treatment of hydrocephalus. The procedure is relatively simple, but there are several potential complications including intracerebral hemorrhage, shunt infection, malfunction, slit ventricle, and subdural hematoma. ${ }^{1)}$ Here we report a case of cerebral venous thrombosis (CVT) after VPS. This is the first reported case of CVT after VPS where radiological study clearly demonstrated the thrombus formation just adjacent to the shunt tube.

\section{Case Report}

A 69-year-old right-handed man, who had no remarkable past history, suffered from bradykinesia, gait, and memory disturbances for 4 years. Computed tomography (CT) and magnetic resonance imaging (MRI) showed an enlargement of the bilateral ventricles (Fig. 1A). His gait was awkward and anteverted. Routine blood cell, chemistry and cerebrospinal fluid (CSF) examinations

Received September 8, 2012; Accepted March 14, 2013 revealed no abnormalities. The lumbar puncture revealed CSF pressure of $160 \mathrm{mmH}_{2} \mathrm{O}$. The tap test was judged as positive. Radionuclide cisternography using diethylenetriamine pentaacetic acid revealed early ventricular reflux and delayed clearance, and thus we diagnosed the patient with idiopathic normal pressure hydrocephalus.

Because the right ventricle was smaller than the left one (asymmetry of the bilateral ventricles), a VPS was performed on the left side using a Codman ${ }^{\circledR}$ Hakim ${ }^{\circledR}$ (Johnson \& Johnson Co., Raynham, Massachusetts, USA) programmable valve with a limiting pressure of $120 \mathrm{mmH}_{2} \mathrm{O}$. A burr hole was placed on the left precoronal region, and no cortical vein was found in the operative field. There were no complications encountered during the perioperative period. The patient made a remarkable improvement postoperatively. CT taken 14 days after the surgery showed a decrement of the size of the lateral ventricles. Bilateral subdural effusion also appeared (Fig. 1B). However, we maintained the valve pressure because of the patient's clinical amelioration.

Two months after his VPS surgery, the patient developed convulsive seizures and right hemiparesis. CT showed a brain edema with petechial hemorrhage in the left frontal lobe (Fig. 1C, D). MRI showed progression of the size of 


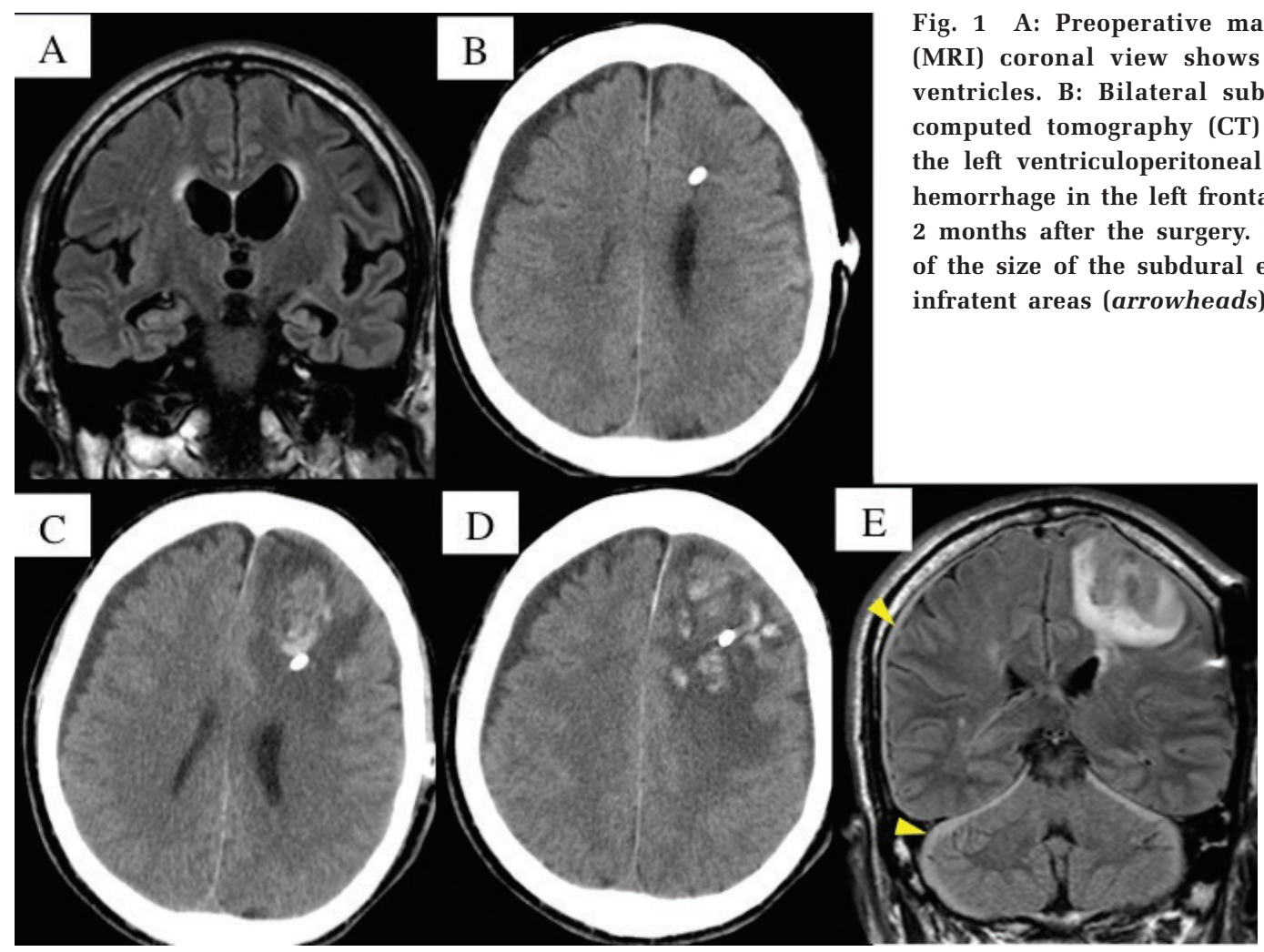

the subdural effusion in both supra and infratent areas (Fig. 1E). MR venography (Fig. 2A, B) showed poor visualization of the superior sagittal sinus and the absence of one of the frontal cortical veins on the left side. A surface image reconstructed from noncontrast CT (Fig. 2C) and the CT coronal view (Fig. 2D) clearly demonstrated the thrombus formation in the cortical vein just adjacent to the shunt tube. We diagnosed this condition as CVT with subsequent venous hemorrhage.

Blood examination on admission revealed a decrement in protein $\mathrm{C}$ activity and antigen $(40 \%$ and $41 \%$, respectively; normal ranges $73-135 \%$ and $62-131 \%$, respectively). Protein $\mathrm{S}$ activity and antigen were decreased to $62 \%$ and $84 \%$ ( $74-132 \%$ and $65-135 \%$, respectively). A coagulation study revealed APTT $40 \mathrm{sec}(28-40 \mathrm{sec})$, PT-INR 1.1 (0.9-1.2), and fibrinogen $300 \mathrm{mg} / \mathrm{dL}$ (150-450 $\mathrm{mg} / \mathrm{dL}$ ). There were no signs of infection. Considering the possibility of intracranial hypotension (IH), we increased the shunt valve pressure to $140 \mathrm{mmH}_{2} \mathrm{O}$ and started anticoagulation therapy with heparin and warfarin. The CVT showed no further aggravation, and the patient's symptoms gradually improved to the point where he was able to resume his normal daily life with some help to deal with his slight right hemiparesis (manual muscle testing 4), gait needing a rollator, and slight disorientation. The protein C level one month after his discharge was $42 \%$ (activity), 39\% (antigen), and the protein $\mathrm{S}$ level was $23 \%$ (activity), 66\% (antigen).

\section{Discussion}

The case described here suggests a possible association between VPS and CVT. To the best of our knowledge, CVT after VPS has not been previously reported. This case provides the impetus for three clinical suggestions.

First, we must be cautious about the tube placement in VPS. In the present case, the CVT and venous hemorrhage occurred 2 months after VPS, and thus the possibility of a direct injury to the cortical vein during surgery is reasonably denied. The tube was initially placed away from the cortical vein during surgery, but the radiological study clearly revealed an acute thrombosed vein located just adjacent to the shunt tube. Noncontrast CT in Fig. 2C and $\mathrm{D}$ demonstrated thrombosed vein from the puncture point of the shunt tube to the superior sagittal sinus, but the magnetic resonance venography (MRV) in Fig. 2A and B did not show it (yellow arrowheads; absence of one of the frontal cortical veins). Hyperdensity of a cortical vein or dural sinus is the primary sign of acute CVT on noncontrast $\mathrm{CT}^{2}{ }^{2)}$ Thus in this case, CT and MRV demonstrated an acute thrombosed vein just adjacent to the shunt tube. We hypothesize that the surface of the brain was shifted by CSF drainage, and the cortical vein was then placed adjacent to the shunt tube. Although there is no direct evidence of compression by the shunt tube or its surrounding tissue, the compression might have occurred to prohibit smooth blood flow, resulting in 


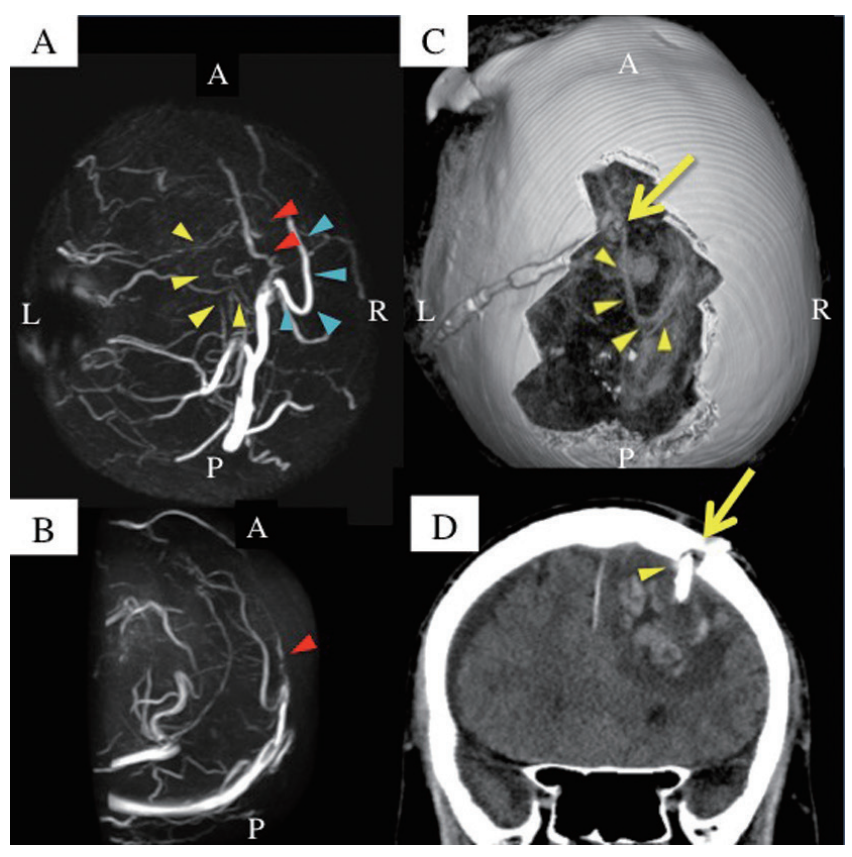

Fig. 2 A, B: Magnetic resonance venography (MRV) shows poor visualization of the superior sagittal sinus (red arrowheads) and the absence of one of the frontal cortical veins on the left side (yellow arrowheads). The frontal cortical vein on the opposite side is marked by blue arrowheads. C: Computed tomography (CT) surface image, the same view as in the MR venography in panel A. This shows the thrombus formation in the frontal cortical vein (arrowheads) from the puncture point (arrow) of the shunt tube to the superior sagittal sinus. D: CT coronal view shows thrombus formation (arrowhead) adjacent to the shunt tube and the puncture point (arrow) of the shunt tube. A: anterior, L: left, P: posterior, R: right.

thrombus formation in this patient. Therefore, we have to make sure that the shunt tube should be placed as far as possible from the cortical veins in consideration of brain shift, even though the cortical vein was not seen through the burr hole during the operation.

Second, the brain shift might have been caused by IH. IH or an imbalance in the CSF pressure gradient may cause CVT., ${ }^{1,3}$ ) In our patient, the CT and MRI findings of progressing subdural effusion suggest CSF over-drainage, which may have led to $\mathrm{IH}$, although we did not measure the patient's CSF pressure. This leads us to assume an analogy between the present case and a post-dural-puncture patient regarding the mechanism of CVT formation. ${ }^{8-15)}$ After dural puncture, CSF leakage leads to IH and decreased CSF. A "rostrocaudal sagging" effect acts on the intracranial contents. A negative spinalcranial pressure gradient and an abnormal pressure gradient between the cerebral vasculature and the CSF space may stretch, dilate, and/or damage the cerebral veins, leading to thrombus formation. ${ }^{16)}$ In our patient, VPS over-drainage may have led to decreased CSF, as may occur with post-dural puncture. In addition, there may have been adhesion in the surgical site in our patient, and thus the surgical adhesion and the change in CSF pressure might have resulted in stretching and tensing of the cortical vein, leading to thrombus formation. Surgeons should, therefore, be cautious about the maintenance of adequate CSF pressure.

Third, closer vigilance is needed after VPS in patients with thrombophilic diathesis. Our patient had low levels of protein $\mathrm{C}$ activity and antigen, which may have been associated with CVT. Previous reports indicate that most patients with post-dural-puncture CVT had a thrombophilic condition, such as the use of oral contraceptives or corticosteroids, factor V Leiden abnormalities, or hereditary protein S or C deficiency. ${ }^{16,17)}$ Because our patient had no other personal or family history of thrombus, we did not perform genetic testing.

Although the above three reasons for the occurrence of CVT are plausible, we cannot deny the possibility that the CVT in the present case may have been a coincidence and thus not related to the VPS. Nevertheless, the close contact of the cortical vein with the shunt tube strongly suggests the following series of phenomena: over-drainage, brain shift, compression, and/or tension of the cortical vein and thrombus formation.

It is not certain whether lumboperitoneal shuntig (LPS) could avoid CVT in this patient. There are no reports comparing the incidence of CVT between LPS and VPS. Because this report first described the radiologically confirmed VPS-associated CVT, further study is needed to compare the incidence of CVT between LPS and VPS.

In conclusion, we report here the first case of CVT after VPS where radiological study clearly demonstrated the thrombus formation just adjacent to the shunt tube. When performing VPS, we must be cautious about the tube placement and the maintenance of adequate CSF pressure. Closer vigilance may be needed for patients with known thrombophilic conditions. Although further studies are needed to confirm the present propositions, we at least should be aware that CVT can occur after VPS.

\section{Conflicts of Interest Disclosure}

The authors have no personal, financial, or institutional interest in any of the drugs, materials, or devices in the article. All authors who are members of The Japan Neurosurgical Society (JNS) have registered online Selfreported COI Disclosure Statement Forms through the website for JNS members.

\section{References}

1) Browd SR, Gottfried ON, Ragel BT, Kestle JR: Failure of cerebrospinal fluid shunts: part II: overdrainage, loculation, 
and abdominal complications. Pediatr Neurol 34: 171-176, 2006

2) Saposnik G, Barinagarrementeria F, Brown RD, Bushnell CD, Cucchiara B, Cushman M, deVeber G, Ferro JM, Tsai FY, American Heart Association Stroke Council and the Council on Epidemiology and Prevention: Diagnosis and management of cerebral venous thrombosis: a statement for healthcare professionals from the American Heart Association/American Stroke Association. Stroke 42: 1158-1192, 2011

3) Albayram S, Kara B, Ipek H, Ozbayrak M, Kantarci F: Isolated cortical venous thrombosis associated with intracranial hypotension syndrome. Headache 49: 916-919, 2009

4) Albayram S, Tasmali KM, Gunduz A: Can spontaneous intracranial hypotension cause venous sinus thrombosis? J Headache Pain 8: 200-201, 2007

5) Berroir S, Grabli D, Héran F, Bakouche P, Bousser MG: Cerebral sinus venous thrombosis in two patients with spontaneous intracranial hypotension. Cerebrovasc Dis 17: 9-12, 2004

6) Lai PH, Li JY, Lo YK, Wu MT, Liang HL, Chen CK: A case of spontaneous intracranial hypotension complicated by isolated cortical vein thrombosis and cerebral venous infarction. Cephalagia 27: 87-90, 2007

7) Sopelana D, Marcos A, Arroyo R, Gutiérrez E, Cuenca R, Vázquez AV, González JL, Egido JA: May intracranial hypotension be a cause of venous sinus thrombosis? Eur Neurol 51: 113-115, 2004

8) Aidi S, Chaunu MP, Biousse V, Bousser MG: Changing pattern of headache pointing to cerebral venous thrombosis after lumbar puncture and intravenous high-dose corticosteroids. Headache 39: 559-564, 1999

9) Canhão P, Batista P, Falcão F: Lumbar puncture and dural sinus thrombosis-a causal or casual association? Cerebrovasc Dis 19: 53-56, 2005

10) Ghatge S, Uppugonduri S, Kamarzaman Z: Cerebral venous sinus thrombosis following accidental dural puncture and epidural blood patch. Int J Obstet Anesth 17: 267-270, 2008

11) Kueper M, Goericke SL, Kastrup O: Cerebral venous thrombosis after epidural blood patch: coincidence or causal relation? A case report and review of the literature. Cephalagia 28: 769-773, 2008

12) Miglis MG, Levine $\mathrm{DN}$ : Intracranial venous thrombosis after placement of a lumbar drain. Neurocrit Care 12: 83-87, 2010

13) Mouraux A, Gille M, Dorban S, Peeters A: Cortical venous thrombosis after lumbar puncture. J Neurol 249: 1313-1315, 2002

14) Sinha A, Petkov S, Meldrum D: Unrecognised dural puncture resulting in subdural hygroma and cortical vein thrombosis. Anaesthesia 65: 70-73, 2010

15) Yildiz OK, Balaban H, Cil G, Oztoprak I, Bolayir E, Topaktas S: Isolated cortical vein thrombosis after epidural anesthesia: report of three cases. Int J Neurosci 120: 447-450, 2010

16) Milhaud D, Heroum C, Charif M, Saulnier P, Pages M, Blard JM: Dural puncture and corticotherapy as risk factors for cerebral venous sinus thrombosis. Eur J Neurol 7: 123-124, 2000

17) Martinelli I, Landi G, Merati G, Cella R, Tosetto A, Mannucci PM: Factor $\mathrm{V}$ gene mutation is a risk factor for cerebral venous thrombosis. Thromb Haemost 75: 393-394, 1996

Address reprint requests to: Satoshi Ayuzawa, MD, PhD, Department of Neurosurgery, Graduate School of Comprehensive Human Sciences, University of Tsukuba, Tsukuba, Ibaraki 305-8575, Japan.

e-mail: sayuzawa@k.tsukuba-tech.ac.jp 